Department of Pharmacology, University of Michigan, Ann Arbor

\title{
Differential Effects of Phenobarbital, Pentobarbital and Diphenylhydantoin on Motor Cortical and Reticular Thresholds in the Rhesus Monkey***
}

By

\section{Roy Aston and Eoward F. Domino}

With 5 Figures in the Text

(Received January 13, 1961)

In 1931, KELLER and FuLToN described the action of various drugs on the motor cortex of monkeys. These authors concluded that pentobarbital sodium, in doses which produced light anesthesia, had little effect upon the motor cortex, whereas phenobarbital sodium, administered in equipotent anesthetic doses, abolished motor cortical responses. Subsequently, MERrTT and PUTNAM (1938) found that phenobarbital markedly increased the convalsive threshold for electroshock in cats in non-anesthetic doses, while pentobarbital had little effect except in doses which produced profound drowsiness. Diphenylhydantoin was shown to possess greater anticonvulsant but less soporific effects than phenobarbital. The differential effect of the two barbiturates upon cortical excitability and wakefulness, has been quoted as the basis for the usefulness of phenobarbital in the treatment of grand mal epilepsy (Goodman and Gilman 1955).

The present investigation was undertaken to re-evaluate the previous work of KeLcer and Futron more quantitatively using techniques involving the response of monkeys with chronieally implanted electrodes to electrical stimulation of motor cortical and mesencephalie reticular sites.

\section{Methods}

Five Macaca mulatta monkeys, of either sex, and weighing from 2 to $4 \mathrm{~kg}$ had chronically indwelling stainless steel bipolar electrodes implanted in the mesencephalic reticular formation and, subdurally, on the leg area of motor cortex. Four of these animals also had electrodes placed in the hippocampus. During surgery the animals were anesthetized with pentobarbital. Stereotaxic coordinates for implantation sites

* Supported by grant R-14-69C from the United Cerebral Palsy Research and Educational Foundation, Ine.

**A preliminary report of this study was presented before the American Society for Pharmacology and Experimental Therapentics Meetings in Chicago (Aston and Domno 1960). 
were determined by reference to OLszEwski (1952). The electrodes were fashioned and implanted on the left side according to the method outlined by Domivo and UEKI (1960). A Cannon plug of 25 contacts was held in a modified "Texas Tower tripod plate. About 300000 units of procaine penicillin were administered to the animals upon completion of the operation and repeated daily for 3 to 4 days. No appreciable infection was noted during a period of 9 months after surgery. All animals were maintained on Purina monkey chow and vitamin supplements.

The implanted areas were stimulated electrically using a Grass stimulator beginning about 1 month after surgery. During the experimental periods the monkeys were isolated in a room separate from the investigator, in which the animals could be observed through a one-way window. The animals were restrained in a "Walter Reed" type chair. The parameters of electrical stimulation were for motor cortex: $60 \mathrm{cps}$ with a pulse width of $1 \mathrm{msec}$; for reticular formation: $300 \mathrm{cps}$ and a pulse width of $0.5 \mathrm{msec}$; and for hippocampus: $100 \mathrm{cps}$ with a pulse width of $0.5 \mathrm{msec}$. In all cases the duration of stimulation was 5 sec. Control thresholds were determined for the three areas under study by stimulating the implantation sites at low voltages and increasing the voltage in steps of $0.2 \mathrm{~V}$ until an arbitrarily predefined endpoint was attained. Usually the current strength of stimulation was also determined simultaneously with an oscilloscope. A period of at least 5 min was allowed to elapse between any two successive stimulations.

After obtaining control thresholds various doses of pentobarbital, phenobarbital and diphenylhydantoin, as the sodium salts, were administered intravenously to the animals, and the absolute increase in the electrical threshold for motor, reticular and hippocampal areas was determined. The barbiturates were dissolved in distilled water, and diphenylhydantoin was solubilized in a small volume of $0.1 \mathrm{~N}$ sodium hydroxide and subsequently diluted with distilled water to provide a $\mathrm{pH}$ of 10.7. Controls for these solvents were included in the study. A single dose of pentobarbital or diphenylhydantoin was injected during one experiment and voltage thresholds determined. The time elapsing between the injection of a drug and the final stimulation in any one experiment varied from 1 to 2 hours with pentobarbital, $5 \mathrm{~min}$ being allowed for the onset of drug action, and from 2 to 3 hours with diphenylhydantoin, with 1 hour being allowed for the onset of the drug's effect. Phenobarbital was administered cumulatively with a period of 10 hours between the initial injection and the end of the experiment. After each injection of phenobarbital, 1 hour was allowed for the effect of the drug to develop. In addition to these studies, the dose of barbiturate required to produce loss of both the righting and corneal reflexes in $50 \%$ of 
monkeys with and without electrode implants was determined as an estimate of the anesthetic AD50. In all cases, at least one week was allowed to elapse between successive drug treatments in any one animal. Doses of drugs employed were $5,10,15,20$ and $25 \mathrm{mg} / \mathrm{kg}$ of pentobarbital, 40,60 and $80 \mathrm{mg} / \mathrm{kg}$ phenobarbital and $10,20,30$ and $40 \mathrm{mg} / \mathrm{kg}$ for diphenylhydantoin. In the case of the two barbiturates a zero dose refers to control thresholds in the untreated animal. In the case of diphenylhydantoin, the zero dose refers to the response of the monkeys to control injections of solutions of sodium hydroxide adjusted to $\mathrm{pH} 10.7$.

The experimental design used for the study of the two barbiturates was a randomized block, blocks being equated to monkeys. In the case of diphenylhydantoin, a 5 by 5 Latin square design was employed consisting of monkeys, doses and time intervals. Analyses of variance and regression analyses of the data were done according to methods outlined by BuRN et al. (1950).

All electrode sites were confirmed histologically using the Hess iron deposition technique and thionin counterstaining as modified by DoMINO (1955).

\section{Results}

Motor cortex. The endpoint for stimulation of the motor cortex was a flexion, usually of the right leg, followed by minimal clonic activity. Control thresholds over a period of 8 months were very reproducible. The mean threshold $\pm \mathrm{SE}$ for 82 observations in the 5 monkeys was $3.2 \pm 0.02 \mathrm{~V}$. The effect of intravenous administration of pentobarbital upon the threshold for motor cortical stimulation is illustrated in Fig. 1. In this figure, as in all figures involving responses to barbiturates, each. of the five values for control threshold represents a mean of 5 determinations in each monkey, since control thresholds were recorded immediately prior to each injection of pentobarbital. Every other point in this, and succeeding figures, represents a single threshold determination in one animal. Figs. 2 and 3 illustrate the regression lines for phenobarbital and diphenylhydantoin respectively. In all three cases the equation of the line and its approximate $95 \%$ limits are included. It is of interest to note that, as calculated by "STUDENT's" $t$-test, the regression coefficients for these three agents are all significantly different one from another $(P<0.05)$.

When the results of the studies with the two barbiturates were subjected to analysis of variance, it was found that significant differences $(<0.05)$ occurred between both monkeys and doses, and that for both drugs, the slope of the regression of absolute voltage on dose was significant with no significant deviation from linearity. For diphenylhydantoin, in which the five monkeys were given 5 doses each (including a 
sodium hydroxide control) during 5 separate experimental time intervals, it was found, by analysis of variance, that both the animals and the doses, exhibited significant differences among them. selves, but that no sig. nificant differences occurred among the responses of any one monkey over the $5 \mathrm{ex}$ perimental intervals $(P<0.05)$. In the case of this drug, also, the regression of voltage threshold on dose was not significantly nonlinear.

From the regression equations, estimates of the potency of the three agents were calculated as the dose required to elevate the threshold by $50 \%$ above the control level. These estimates of "TD 50 " are $10.8,19.9$ and $35.5 \mathrm{mg} / \mathrm{kg}$ for pentobarbital,phenobarbital and diphenylhydantoin, respectively. Expressed as potency ratios, this means that, in terms of the effect in elevating motor cortical thresholds by $50 \%$, pentobarbital was $\mathbf{3 . 3}$ times as potent as diphenylhydantoin, while phenobarbital was only 1.8 times as potent. Of the two barbiturates,

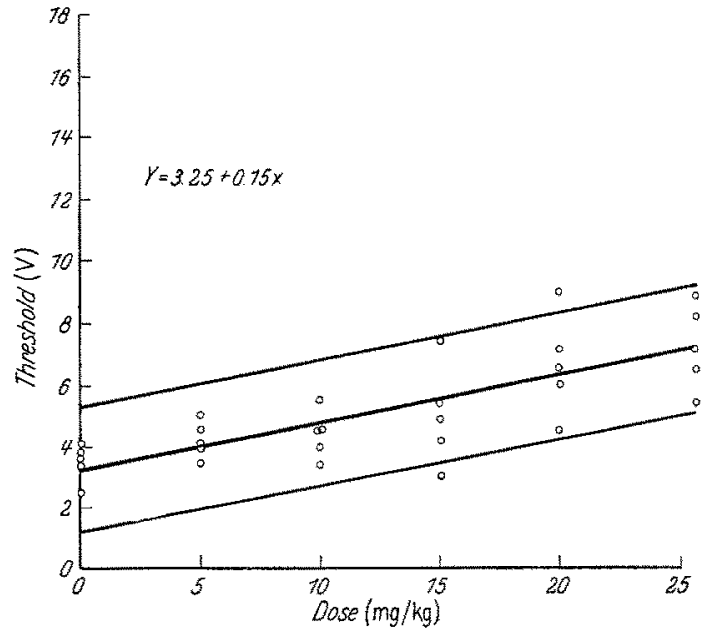

Fig. 1. Regression line and equation of the effect of pentobarbital upon the threshold for electrical stimulation of the motor cortex. In this and all subsequent figures the equation. of the regression line of voltage threshold against drug dose in $\mathrm{mg} / \mathrm{kg}$ given intravenously is shown. The individual points, the regression line and its $95 \%$ eonfidence limits appear as small circles, a thick and two thin lines, respectively

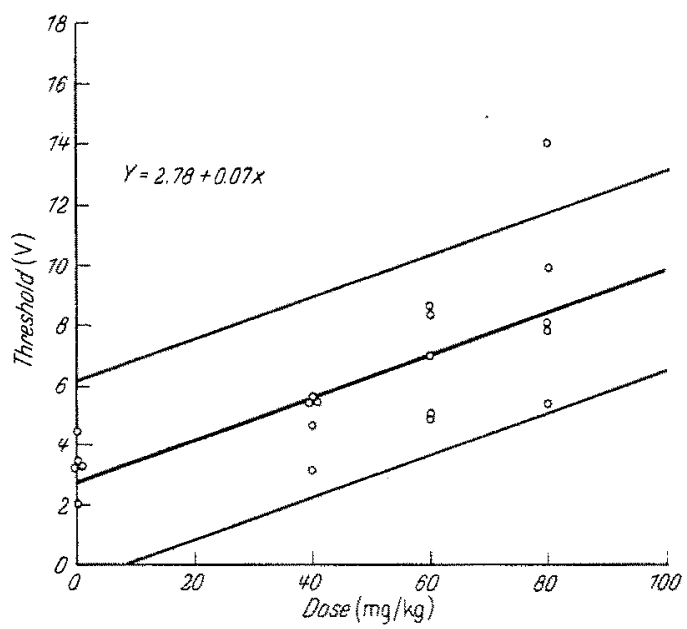

Fig. 2. Regression line and equation of the effect of phenobarbital upon the threshold for electrical stimulation of the motor cortex

pentobarbital was 1.8 times as potent as phenobarbital in elevating the motor cortical threshold. 
The pattern of motor seizures after the administration of each of these agents differed. Although pentobarbital raised the threshold

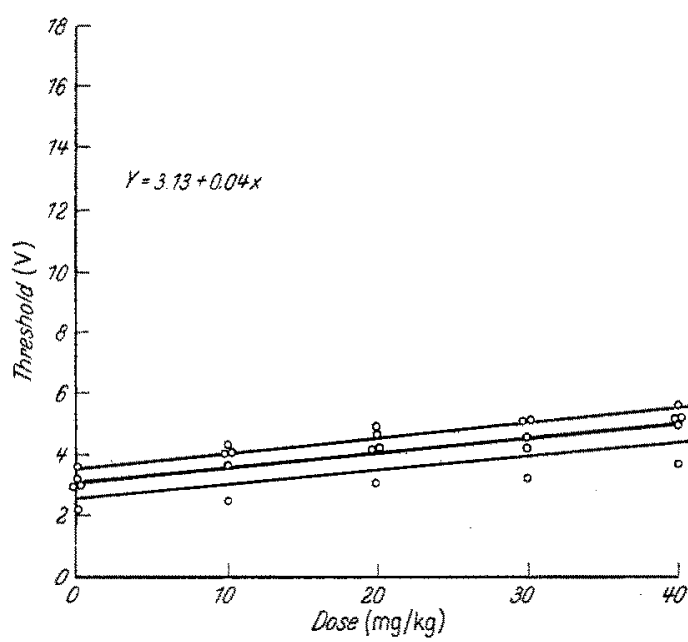

Fig. 3. Regression line and equation of the effect of diphenylhydantoin upon the threshold for electrical stimulation of the motor cortex

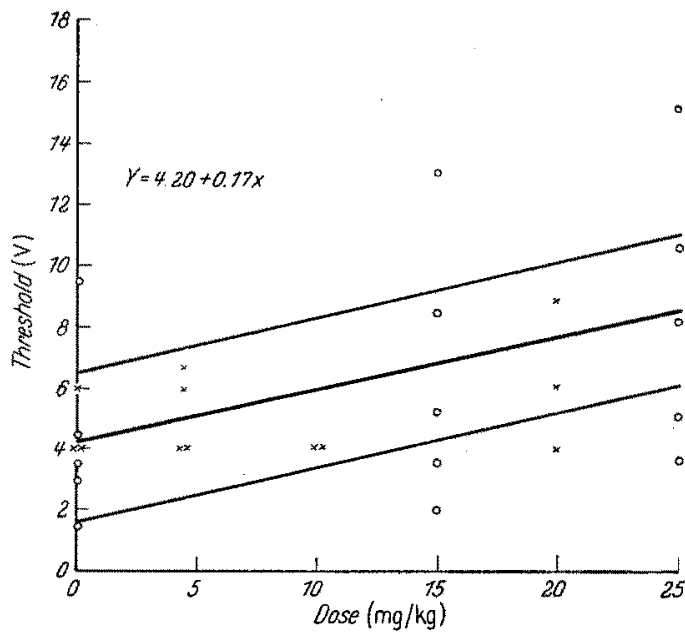

Fig. 4. Regression line and equation of the effect of pentobarbital upon the threshold for electrical stimulation of the mesencephalic reticular formation. The o points represent all five monkeys given different doses. The $x$ points represent the data of varions monkeys given intermediate doses for motor seizures, it had little effect on seizure duration. Before drug treatment in doses of 5 to $20 \mathrm{mg} / \mathrm{kg}$ the mean duration was $19.2 \mathrm{sec}$ and after 14.0 sec. This decrease was not significant by the $t$-test $\quad(P<0.10)$. After either phenobarbital or diphenylhydantoin the period of clonus was verymuch shortened or abolished so that the total period of motor movement at threshold was reduced to the duration of electrical stimulation, i.e. 5 sec. Even increasing the stimulus voltage considerably above threshold failed to prolong the duration of flexion.

Reticular formation. Two distinct endpoints were chosen for reticular stimulation. No stimulation was given until an animal had lost all behavioral signs of agitation and was sitting quietly. Those animals which had received the larger doses of barbiturates, of course, lapsed into drowsy or sleeping states between stimulations. One endpoint, or "minimal motor response", ineluded behavioral alerting and opening wide of the palpebral fissures, generally together with tightening of the 
scalp and wiggling of the ears, slight flexion of one leg or some turning of the head and shoulders to the ipsilateral side. As the voltage was slowly increased the motor signs became more exaggerated and vocalization occurred. This second, or "squawk response", was typically manifested by flexion of the arm and opening of the mouth contralaterally, flexion of the leg at the hip ipsilaterally, and flattening of the ears against the head, together with a stertorous hyperpnea at subthreshold voltages which developed into a high pitched squawk as the stimulus voltage was increased. The mean control thre shold \pm SE for 49 observations over 8 months in the 5 animals was $4.9 \pm 0.37 \mathrm{~V}$ for "minimal motor response" and $6.5 \pm 0.25 \mathrm{~V}$ for the "squawk response".

The control threshold for the minimal motor response was increased by the two barbiturates employed, but not by diphenylhydantoin. Figs. 4 and 5 illustrate the regression of voltage threshold upon dose

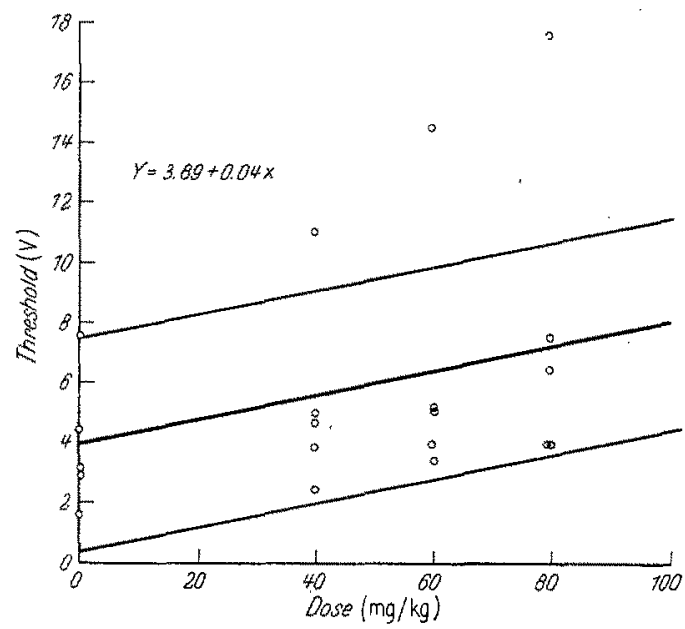

Fig. 5. Regression line and equation of the effect of phenobarbital upon the threshold for electrical stimulation of the mesencephalic reticular formation

for pentobarbital and phenobarbital, respectively. The corresponding regression coefficients, 0.17 and 0.04 , are significantly different $(P<0.01)$. The slopes for the regression lines relating threshold and dose for pentobarbital in its effect upon motor cortical and reticular areas are, however, homogeneous, as are the slopes for phenobarbital in these two cases $(P<0.10)$. The doses of barbiturate required to raise the voltage threshold for the reticular motor response by $50 \%$ was $12.4 \mathrm{mg} / \mathrm{kg}$ for pentobarbital and $48.6 \mathrm{mg} / \mathrm{kg}$ for phenobarbital. Thus pentobarbital was 3.6 times as potent as phenobarbital in elevating the reticular threshold for minimal motor response.

Analysis of variance for the "squawk response" to reticular stimulation showed that none of the three agents employed significantly altered the threshold for this phenomenon. Since the voltages required to elicit the "squawk response" were quite high, the phenomenon appeared to be related to supramaximal stimuli and current spread to extrareticular pathways. 
Histological examination showed that reticular electrodes were placed at the border between the mesencephalic tectum and tegmentum at the level of the upper border of central grey. Frequently the upper stimulating electrode bordered the superior or inferior colliculus and occasionally was in it.

Anterior hippocampus. The endpoint for stimulation of the hippocampus was taken to be a change in hippocampal EEG activity, which was recorded on an 8-channel ink-writing electroencephalograph. Hippocampal afterdischarge resembled high voltage, hypersynchronized spikelike activity. The mean control threshold \pm SE for 53 observations over 8 months in 3 monkeys was $8.0 \pm 0.26 \mathrm{~V}$. Only 3 of the 4 implanted monkeys manifested hippocampal afterdischarge in response to stimulation. During the period of electrical stimulation of the hippocampus and its subsequent afterdischarge minimal gross motor activity was observed in three of the monkeys. Stimulation of the "hippocampal" area in the fourth monkey elicited a ptosis of the right eye with tremor of the right eyelid and a turning of the eyes to the extreme right. The head was also turned to the unimplanted side. This response was seen at about $6 \mathrm{~V}$. When the stimulus strength was increased to about $8.0 \mathrm{~V}, 3$ to $5 \mathrm{cps}$ spike and dome seizure activity was produced in Area 17 with high voltage waves of the same frequency occurring in the hippocampus and sometimes in Area 1 and reticular formation which lasted 25 - 35 sec. Upon sacrificing this animal one of the "hippocampal" electrodes was found to be located in the lateral geniculate and the other at the superior border of the hippocampus. In the other animals the electrodes were within or bordering the inferior or lateral aspect of the anterior hippocampus.

After all three drugs, the hippocampal threshold showed an elevation in proportion to dose. However, in no case did analysis of variance reveal a significant difference between doses $(P>0.05)$, perhaps due to an insufficient number of trials. In estimating the approximate doses required to elevate the hippocampal threshold by $33 \%$, however, it appeared that phenobarbital was the least potent of the three agents used, while pentobarbital was approximately 3.3 times, and dipbenylhydantoin 6.2 times as potent.

Anesthetic effects. The dose of intravenously administered barbiturate required to anesthetize $50 \%$ of treated monkeys (AD50) was estimated for pentobarbital and phenobarbital in normal, unimplanted monkeys, and for pentobarbital in implanted monkeys. Five to eight animals were used for each estimate of AD 50, this estimate being based on 29 to 30 observations for each treatment. The endpoint chosen as an index of anesthesia was the loss of both righting and corneal reflexes. The results were subjected to probit analysis. In all cases analysis of 
variance showed the regression of probit upon log dose to possess a signifieant slope $(P<0.01)$ and to possess no significant deviation from linearity. The table lists the regression equations and the $\mathrm{AD} 50 \pm \mathrm{SE}$ with its $95 \%$ confidence limits for each of three treatments. The slopes for the regression lines of the two barbiturates in the unimplanted monkey, 63.64 and 39.46 were not significantly different $(0.50>P>0.10)$, as judged by the $t$-test. Each of these two slopes differs significantly from that of the regression line for pentobarbital in the implanted monkey $(P<0.01)$. The low value of the latter slope, 5.19 , indicates that anesthetic thresholds in the implanted animals vary more widely than those in unoperated monkeys.

The gross effects of diphenylhydantoin in the monkey were negligible at 10 and $20 \mathrm{mg} / \mathrm{kg}$. At $40 \mathrm{mg} / \mathrm{kg}$ the animals showed licking, mucoid salivation, chewing and gagging as well as mydriasis and vertical nystagmus immediately upon injection. An apparent hindlimb muscular weakness also developed which was manifested as an ataxia when the animal was freed in its cage. The nystagmus and particularly the ataxia were evident for several hours after injection. In contrast to the barbiturates, no evidence of deep sedation was seen at this dose level.

Correlation of voltage and milliamperage. Current strength of stimulation was monitored during the major part of this study. However, all threshold determinations were reported in this manuseript in terms of voltage. In order to determine whether this procedure had adversely influenced the data, coefficients of correlation between voltage and milliamperage were determined.

Voltage and milliamperage data were collected for each monkey, from experiments upon both motor and reticular areas over a period of 6 months, providing two estimates of correlation. In 5 monkeys so considered, a total of 10 correlations was determined, ranging from +0.886 to +0.9998 , each of which was statistically significant $(P<$ 0.001 ), with a mean of +0.969 . Also, the extent of correlation between voltage and milliamperage was estimated from 100 observations (20 from each animal) collected over a period of 8 months, with observations representing studies of motor, reticular and hippocampal areas. This provided a correlation of +0.922 . As might be expected, voltage and milliamperage was much better correlated within one area of one monkey than when all implanted areas were included in the calculation. However, the difference was not statistically significant.

\section{Discussion}

Pentobarbital, phenobarbital and diphenylhydantoin were all effective in elevating motor cortical thresholds. Since the regression coefficients for the dose-response curves of these agents were statistically heterogeneous, relative potencies of these agents could not be expressed 
as a single figure. If one chose an arbitrary endpoint of a $50 \%$ increase in voltage threshold it is evident that pentobarbital was the most potent of the three drugs employed, being 1.8 times as potent as phenobarbital and 3.3 times as potent as diphenylhydantoin. It is of interest that although pentobarbital was the most potent of the three agents studied in elevating motor cortical threshold, it did not appear to alter the duration or the character of the convulsive seizure obtained at threshold voltages. Phenobarbital and diphenylhydantoin in contrast both markedly reduced the clonic component of the induced seizure and shortened its duration. These differences between the two barbiturates together with the finding that the slopes of the regression of voltage threshold on dose differed, suggest that pentobarbital and phenobarbital may increase motor cortical thresholds through different mechanisms.

The observation that phenobarbital raises motor cortical thresholds in monkeys confirms the findings of KaLLer and FuLtow (1931). The results of the present study on motor cortical thresholds also agree in principle with those obtained by DELGado and MrdaILovió (1956) in the monkey, except that, in the latter investigation diphenylhydantoin was found to be more potent in increasing the threshold for motor afterdischarge than phenobarbital. On the other hand, GANGLOFF and MonNIER (1957), working with the rabbit, concluded that diphenylhydantoin affected neither the threshold nor the duration of electricallyinduced cortical afterdischarge, and that phenobarbital actually reduced this threshold while having no effect upon duration. Such responses might have been due to very low blood levels of the drugs used since, although these authors administered up to $150 \mathrm{mg} / \mathrm{kg}$ of diphenylhydantoin and up to $60 \mathrm{mg} / \mathrm{kg}$ of the barbiturate, both agents were given by mouth. In the rabbit, special dietary precautions must be taken to ensure emptying of the stomach (Markowitz 1954), and it is conceivable that the animals employed by GANGLOFF and MoNNIER carried undigested food in their alimentary tracts, reducing the rate of absorption of the orally-administered drugs. Furthermore, it has been shown by SCHÜTZ and CASPERS (1953) that with mild depression of the central nervous system, there is an increased tendency to cortical seizures in rats with experimental epileptogenic foci. A low absorption rate of phenobarbital might thus explain the reduced threshold for cortical activation observed by GANGLOFF and MonNrER. It is also well known that low doses of various barbiturates, particularly in rodents such as the mouse, produce marked initial motor stimulation. This is less true of the rabbit. Thus, the former arguments appear less convincing, particularly in view of the fact that GANGLOFF and MoNNIER were able to show that phenobarbital in doses lowering motor cortical thresholds markedly elevated diencephalic and rhinencephalic seizure thresholds. 
Therefore, species differences may account for the discrepancies observed by various investigators.

The gross behavioral responses evoked by reticular stimulation appear to be similar to those noted by FR.ENCH (1958) following stimulation of cortical areas projecting into the reticular formation of the monkey. These responses consisted primarily of alerting at low intensities of stimulation and signs of panic at higher intensities. The associated vocalization which was observed in this study was not reported by French. This is probably due to the fact that the reticular electrodes were high in the mesencephalon and current spread into associated pathways in the superior or inferior colliculi. Both barbiturates raised the threshold for the minimal motor reaction but left the threshold for the "squawk response" unaffected. If phenobarbital is considered a standard, the relative potency of pentobarbital (to cause a $50 \%$ increase in reticular threshold) was 3.92. Neither the motor nor the vocal response to reticular stimulation was affected by diphenylhydantoin in the doses used. These data corroborate those of Martin, Vernater and UNNA (1954) who found that phenobarbital depressed the activating response to reticular stimulation while diphenylhydantoin did not. It has been suggested that the reticular formation is one of the principal sites of action of anesthetic agents (French et al. 1953). According to this hypothesis the present results provide some basis for clinical anticonvulsant effectiveness as described previously. Pentobarbital, although the most potent in its ability to increase motor cortical thresholds, would be useless as a chronic medication for the grand mal epileptic because of its marked sedative effects as exemplified by a ratio of motor cortical TD50 to reticular TD50 of only 1.15. Phenobarbital, on the other hand, manifests a two-fold increase in this ratio of 2.44. Diphenylhydantoin, although the least potent of the compounds studied in terms of motor cortical depressant effects nevertheless had no demonstrable action upon reticular thresholds and therefore showed the greatest ratio. The determination of the anesthetic potencies of the two barbiturates provided the same order of potency as did the studies on reticular thresholds. Pentobarbital was 6.24 times as potent as phenobarbital. In the doses employed, diphenylhydantoin showed no general anesthetic properties. It is obvious that effective elevation of motor cortical thresholds can be accomplished without markedly altering the reactivity of the reticular core to electrical stimulation or producing signifieant anesthesia. These results help explain the observation of MerRitT and Puttiran (1938) that diphenylhydantoin increases electroshock thresholds without producing marked sedative effects. Thus the present investigation provides further scientific rationale for the clinical usefulness of phenobarbital and diphenylhydantoin in grand mal and 
cortical focal (Jacksonian) epilepsy. However, it should be pointed out that the pyramidal system is not crucial to the development of generalized seizures (Gastaut and Fisher-WrLltams 1959). Thus electrical stimulation of the motor cortex may in no way mimic the site of origin of grand mal epilepsy. In fact there is evidence of many important subcortical mechanisms in such seizures. Nevertheless, electrical stimulation of the motor cortex is a very convenient means of initiating abnormal seizure discharge in the experimental animal.

In the present study all three drugs employed showed a tendency toward elevation of the voltage threshold for electrical afterdischarge in the hippocampus. Diphenylhydantoin appeared to be the most potent and phenobarbital the least potent. Both barbiturates were less effective in raising the hippocampal threshold than in raising the cortical threshold. Diphenylhydantoin proved to be more potent in its hippocampal than in its motor cortical effects. The preliminary findings of an elevation in hippocampal thresholds by phenobarbital in monkeys confirms the work of GANGLofF and Monnier (1957) in rabbits. These authors, however, found no effect of diphenylhydantoin on rhinencephalic seizure thresholds. Species differences of inadequate absorption of diphenylhydantoin administered orally might account for this negative finding as described previously.

The results of the anesthetic studies with barbiturates indicate that, in those monkeys which had implanted cerebral electrodes, the variation in response to single doses of the agent was greater than that in unoperated monkeys. This is evident if the standard error of the AD50 is expressed as a percentage of the AD50. If this is done the "\% error" in the unimplanted monkeys is 1.09 and $1.90 \%$ for phenobarbital and pentobarbital respectively, while that for pentobarbital in implanted animals is $15.58 \%$ (see table). Simultaneously solving the regression equations for pentobarbital in the implanted and unimplanted monkeys gives 
the intersection of these two lines at a point corresponding to $16.44 \mathrm{mg} / \mathrm{kg}$ and $50.5 \%$ response. At doses below $16.44 \mathrm{mg} / \mathrm{kg}$ the implanted animals are more susceptible to the effects of the drug, while at doses above $16.44 \mathrm{mg} / \mathrm{kg}$ the implanted animals are more resistant to the drug's effects than the unimplanted ones. The results obtained in this study using low doses are in keeping with the results of SequrN and Stavrakx (1957), Helier et al. (1960), and AdLer (1960) who found that brain lesions including the septal forebrain, frontal cortex or caudate nucleus markedly increase barbiturate sleeping time. It is possible that the anterior hippocampal implantations in the monkeys used in the present study may have had an influence upon septal activity since these two areas are known to possess fibre connections.

The extent of activation of the neuronal mass at the electrode tip is dependent upon the energy supplied by electrical stimulation. Energy imparted by electrical eirenits is directly proportional to the current strength, duration of application and tissue impedance. Since the latter may vary, milliamperage appears to be the parameter of choice for studies of electrical threshold. Both amperage and voltage have been used in previous studies. In the present investigation a significant correlation has been shown to exist between milliamperage and voltage threshold determinations. Thus, if the regression of milliamperage on some variable is linear, the relationship between this variable and voltage will also manifest linearity. In this study the quantification of drug effect upon threshold of stimulation is dependent solely upon the establishment of linear dose-response relationships and, for this reason estimates of voltage threshold were considered adequate.

\section{Summary}

Rhesus monkeys were implanted chronically with bipolar electrodes in motor cortical, mesencephalic reticular and anterior hippoeampal areas. Voltage and current thresholds of stimulation were determined for the elicitation of gross motor seizures, alerting responses, and electrieal seizures, respectively before and after varying doses of pentobarbital, phenobarbital and diphenylhydantoin. Anesthetic potencies were also estimated. Diphenylhydantoin was the most specific in terms of increasing motor cortical thresholds in doses that had no significant effect upon reticular thresholds. Phenobarbital was somewhat less specific than diphenylhydantoin. It elevated motor cortical thresholds in doses which only minimally increased reticular thresh. olds. Pentobarbital increased both motor cortical and reticular thresholds.

The potency in elevating motor cortical thresholds was inversely related to the degree of specificity. Thus, pentobarbital was the most 
potent, phenobarbital second, and diphenylhydantoin least effective in increasing motor cortical thresholds. In elevating the threshold for hippocampal afterdischarge phenobarbital appeared to be the least potent while diphenylhydantoin appeared the most potent.

Anesthetic potencies of these drugs followed the same trend as did their effects on reticular thresholds. Thus, pentobarbital was the most potent, phenobarbital second and diphenylhydantoin was least effective having no anesthetic effects.

Voltage thresholds were found to be significantly correlated with milliamperage thresholds.

It is concluded that this study provides additional scientific rationale for the clinical usefulness of phenobarbital and diphenylhydantoin in the treatment of cortical focal (Jacksonian) and grand mal epilepsy.

\section{References}

AdLer, M.W.: Changes in sensitivity to hexobarbital and pentylenetetrazol after brain lesions in rats. Pharmacologist 2, 76 (1960).

Aston, R., and E.F. Domino: Differential effects of phenobarbital, pentobarbital and diphenylhydantoin on motor cortical and reticular thresholds in monkeys. Fed. Proc. 19, 182 (1960).

BURN, J.H., D. J. FlNNeY and L. G. GoodWIN: Biological standardization, 2nd edit. London: Oxford University Press 1950.

Delgado, J.M.R., and L. Mirailovió: Use of intracerebral electrodes to evaluate drugs that act on the central nervous system. Ann. N.Y. Acad. Sci. 64, 644-666 (1956).

Domrro, E.F.: A pharmacological analysis of the functional relationship between the brainstem arousal and diffuse thalamic projection systems. J. Pharmacol. exp. Ther. 115, 449-463 (1955).

-, and S. UEKI: An analysis of the electrical burst phenomenon in some rhinencephalic structures of the dog and monkey. Electroenceph. clin. Neurophysiol. 12, 635-648 (1960).

French, J.D.: Corticifugal connections with the reticular formation. Chapt. 24, in reticular formation of the brain, J.H. JASPER, L.D. Proctor, R. S. KNIGHTON, W. C. Noshay and R.T. Costello, editors. Boston: Little, Brown \& Co. 1958.

- M. Verzeano and H.W. Magoun: Neural basis of anesthetic state. A.M.A. Arch. Neurol. Psychiat. 69, 519-529 (1953).

GaNGLOFF, H., and M. Monnier: The action of anticonvulsant drugs tested by electrical stimulation of the cortex, diencephalon and rhinencephalon in the unanesthetized rabbit. Electroenceph. clin. Neurophysiol. 9, 43-58 (1957).

Gastaut, H., and M. Fisher-WiLliams: The physiopathology of epileptic seizures. Chapt. 14, in Handbook of physiology, sect. 1, vol. 1, Neurophysiology, FIELdD, JR. edit. Washington, D.C.: American Physiological Society 1959.

Goodman, L.S., and A. GmMan: The pharmacological basis of therapeutics, 2nd edit. New York: MacMillan Company 1955.

Heller, A., J.A. Harvey, H.F. Hunt and L.S. Roth: Effect of lesions in the septal forebrain of the rat on sleeping time under barbiturate. Science 131, $662-664(1960)$. 
Kelcer, A.D., and J.F. Fulton: The action of anesthetic drugs on the motor cortex of monkeys. Amer. J. Physiol. 97, 537 (1931).

Markowitz, J.: Experimental surgery, 3rd edit. Baltimore: Williams \&Wilkins 1954.

Martin, W.R., V.G. Verniter and K.R. Unda: Effects of Dilantin and phenobarbital on the response of the cortex to stimulation of the activating center. J. Pharmacol. exp. Ther. 110, 35 (1954).

Merritt, H.H., and T.J. Putnam: A new series of anticonvulsant drugs tested by experiments on animals. A.M.A. Arch. Neurol. Psychiat. 39, 1103-1015 (1938).

OLsZewski, J.: The thalamus of the Macaca mulatta, 1st edit. Basel: Karger 1952.

SchüтZ, E., and M. CASPERS: On provocation and activation of epileptic discharges by anesthesia and sleep induced by medicaments. Electroenceph. clin. Neurophysiol. 5, 118 (1953).

SequTr, J.J., and G.W. STAVRakY: The effects of barbiturates on partially isolated regions of the central nervous system. Cand. J. Biochem. 35, 667-680 (1957).

Dr. E. F. Domiro, Department of Pharmacology

University of Michigan, Ann Arbor U.S.A.

Dr. Roy Aston, Department of Physiology and Pharmacology

Wayne State University, Detroit U.S.A. 